\title{
Leibniz, Kant, and Referring in the Quantum Domain
}

\section{Cord Friebe ${ }^{1}$}

Published online: 11 July 2020

(C) The Author(s) 2020

\begin{abstract}
The paper addresses the referring problem in quantum mechanics, by spelling out the alternatives with complete or individual concepts (Leibniz; in modern terms: definite descriptive proper names), with directly referential labels (standard formalism of quantum mechanics), and with intuition (Kant). The connection between the way of referring and some metaphysical theses about objects will be explained. Then, the paper intends to make plausible that the Kantian way is the best way for the quantum domain, including quantum field theories.
\end{abstract}

Keywords Leibniz's principle · Quantum mechanics · Proper names · Kant

\author{
Abbreviations \\ QM Quantum mechanics \\ PII Principle of the identity of indiscernibles
}

\section{Introduction}

Leibniz and Kant hold significantly different views about how to refer to spatiotemporal objects, such as drops of water. It goes via complete or individual concepts according to Leibniz, and via intuition according to Kant. Correspondingly, they defend significantly different metaphysical theses: spatiotemporal objects are unique individuals in virtue of (absolutely) qualitative distinguishability versus spatiotemporal objects are merely single particulars individuated by differences in spatial location. ${ }^{1}$

Opposed to both views, the straightforward reading of the standard formalism of QM uses proper names of the directly referential sort in order to refer to physical particles.

\footnotetext{
${ }^{1}$ Throughout the paper with "individuation" is meant synchronic individuation—now or at a given time-, not diachronic individuation, i.e. persistence over time. The notion of an individual is broader than in the current philosophy of physics literature, i.e. not restricted to entities that are absolutely distinguishable; this restriction is historically inadequate and, as it turns out at the end of the paper, also systematically. Concerning "absolute", "qualitative", "haecceitistic", and the like, I intend to follow the terminological stipulations in Caulton and Butterfield (2012).
}

Cord Friebe

cord.friebe@uni-siegen.de

1 Universitat Siegen, Siegen, Germany 
Correspondingly, also the metaphysical theses defended by the majority views in the philosophy of QM differ significantly from both Leibniz's and Kant's views: QM-objects neither are individuated by absolutely qualitatively distinguishing properties nor by spatial locations; they are-perhaps-individuated haecceitistically or are only weakly discernible, i.e. they allegedly are relationals instead of individuals.

Based on the distinction between purely permutation invariant states and physically entangled ones, the two main QM-philosophical views are currently under pressure. ${ }^{2}$ The first way of doing justice to this physical difference is the Leibniz strategy, a defence of the Leibniz package including the PII as the principle of individuation and referring with (comprehensively) descriptive proper names. The second alternative is Kantian. Assuming that the position basis is privileged, individuals arise in virtue of different spatial locations and can be referred to via intuition. The main purpose of the paper is to give a hint why that view should be considered more carefully and why it might be better than the Leibniz strategy.

The structure of the paper is as follows: In Section 2, I will sketch the dialectical landscape spanned by Leibniz, Kant, and the straightforward reading of QM. Section 3 is devoted to the two main views in the philosophy of QM, the Received View and Weak Discernibility. Then, Section 4 presents the Leibniz strategy against these dominant views, based on the deflation of the notion of physical entanglement, according to which (at least) in purely permutation invariant states there in fact are absolutely qualitatively distinguishable particles, i.e. Leibniz-individuals. Finally, in Section 5 I will discuss the Kantian alternative which might turn out to be broader in scope than Leibniz's, applicable also for quantum domains with delocalization and vagueness in particle number.

\section{2 (Dis)agreements Between Leibniz, Kant, and Standard QM}

As I see it, the history of (dis)agreements between Leibniz, Kant, and QM can be told in the following way. ${ }^{3}$ Look at a situation of apparent distinguishability, as presented in Fig. 1.

According to phenomenological prejudice, in this situation there are two qualitatively distinguishable objects. There even are absolutely distinguishable objects: one of the objects is (say) red, the other is green. Leibniz and Kant would agree with the phenomenological prejudice. By contrast, along with the straightforward reading of the standard formalism of QM even in this situation there are two objects that are not absolutely qualitatively distinguishable. For, the QM-state would be unique, as shown in Fig. 2.

Both particle 1 and particle 2 are in the same two-particle state. Concerning each single particle, nothing more can be said than that they are in reduced mixed states, but both particles, labelled by " 1 " and " 2 ", would share their common mixed state, ${ }^{4}$ namely in the case at hand this one:

$$
\hat{\rho}_{1 ; 2}=\frac{1}{2}\left(\left|R, \uparrow_{z}\right\rangle\left\langle\uparrow_{z}, R|+| L, \downarrow_{z}\right\rangle\left\langle\downarrow_{z}, L\right|\right)
$$

\footnotetext{
2 The non-separability of the joint state (of two or more similar particles) is not sufficient for entanglement that rather requires the possibility of violating a Bell inequality. This deflation of the notion of entanglement goes back to Ghirardi et al. (2002).

${ }^{3}$ I assume that everything said in this paper can easily be extended to more than two objects (particles).

${ }^{4}$ Ignorance-interpretation of mixed states not allowed!
} 
Thus, physics disagrees with the phenomenological prejudice already on the pre-philosophical level, whereas Leibniz and Kant on the pre-philosophical level agree with the phenomenological prejudice and so disagree with (the straightforward reading) of QM already on the pre-philosophical level.

Philosophically, Leibniz and Kant have a dispute. According to Leibniz, it is not only actually the case that the two objects are absolutely qualitatively distinguishable, but necessarily so. Kant strikes against Leibniz by claiming that difference in spatial location is sufficient. So, according to Kant the counterfactual situation is possible in which both objects share all their intrinsic properties (by being both red, for instance), whereas according to Leibniz such a situation is impossible. Moreover, according to Kant spatial difference is an "adequate ground" (Kant 1781/81, A 293/B 319) for the numerical distinctness of objects, while according to Leibniz objects are numerically distinct in virtue of absolutely qualitative distinguishability.

Grounding numerical distinctness in absolutely qualitative distinguishability is more than the mere necessity of numerical distinctness going hand in hand with such a distinguishability: it is not only the case that there are no indiscernibles within every possible situation (world; state), but one also must identify indiscernibles across counterfactual situations (worlds; states). This twist can be illustrated by Fig. 3 that shows, apparently, two qualitatively indistinguishable situations.

According to Leibniz, however, one must identify these situations, because one must identify the red object in the first situation with the red object in the second (and the same goes with the green objects). Within each situation, there are absolutely qualitatively distinguishable objects, 'but' qualitatively indistinguishable arrangements have to be counted as one and the same. This is one way-the Leibnizian way-of understanding the requirement of permutation invariance. It is not the QM-way of understanding "permutation invariance".

Applied to QM, the Leibnizian way of understanding "permutation invariance" would mean that non-symmetric product states-e.g., $|R\rangle_{1}\left|\uparrow_{z}\right\rangle_{1}|L\rangle_{2}\left|\downarrow_{z}\right\rangle_{2}$ and $|L\rangle_{1}\left|\downarrow_{z}\right\rangle_{1}|R\rangle_{2}\left|\uparrow_{z}\right\rangle_{2}$ - describe, respectively, each arrangement and, then, somehow have to be identified. However, it is the lesson from French and Redhead (1988, 236-237) that the allowed QM-state (as given in Fig. 2) cannot be obtained by an identification of product states but by a superposition of them which leads to a quite different state. This is again a way of saying that (the standard reading of) QM disagrees with Leibniz (and Kant) from the beginning, namely by rejecting the starting situation as a case of (absolutely) qualitative distinguishability.

Leibniz's way of grounding the numerical distinctness of objects in their (absolutely) qualitative distinguishability is closely connected with Leibniz's philosophy of language doctrine of concepts being complete or "individual". ${ }^{5}$ Individual concepts are like proper names according to descriptivism, the view that names/labels refer to their referents via a definite description of those objects. Take the label "1" in Fig. 3. In one way of understanding labels, " 1 " refers to particle 1 independently of what that particle 'does', i.e. independently of the properties the particle actually possesses. In this way, particle 1 may be red in the first situation but green in the second, and so the qualitatively indistinguishable arrangements must be counted as two. Variants of the theory of direct reference are committed to this reading. According to Leibniz (descriptivism), by contrast, " 1 " refers to the particle that is such-and-such (red, in the case at hand) independently of which that particle

\footnotetext{
5 See Rodriguez-Pereyra (2014, Ch. 4) for the historical reconstruction of how Leibniz derives the PII from his doctrine of complete or individual concepts.
} 
Fig. 1 Two qualitatively distinguishable objects
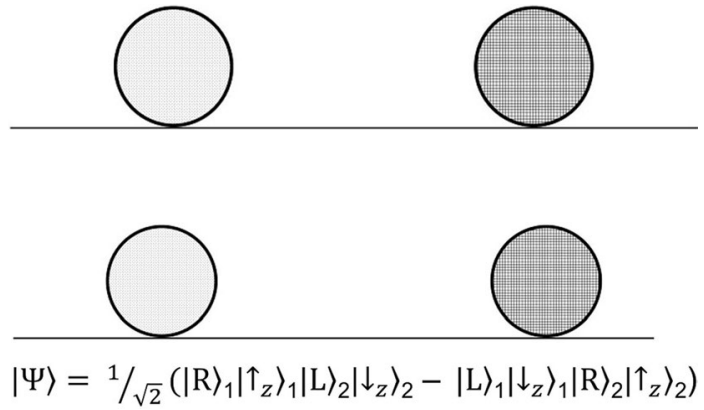

$$
|\Psi\rangle=1 / \sqrt{2}\left(|\mathrm{R}\rangle_{1}\left|\uparrow_{z}\right\rangle_{1}|\mathrm{~L}\rangle_{2}\left|\downarrow_{z}\right\rangle_{2}-|\mathrm{L}\rangle_{1}\left|\downarrow_{z}\right\rangle_{1}|\mathrm{R}\rangle_{2}\left|\uparrow_{z}\right\rangle_{2}\right)
$$

Fig. 2 One single state for both particles

may be. Descriptivism apparently requires to identify, e.g., red objects across possible situations and so is in accordance with Leibniz's way of grounding numerical distinctness in (absolutely) qualitative distinguishability. ${ }^{6}$ The Leibniz package includes a specific way of individuating objects ('PII as principle of individuation'), a specific way of establishing permutation invariance ('identifying indistinguishable arrangements'), and a specific way of using labels ('descriptivism').

The straightforward reading of the standard formalism of QM goes against Leibniz. According to this reading, the tensor product indices " 1 " and " 2 " refer to physical particles but not in the descriptivist's way. Consider the running state from Fig. 2:

$$
|\psi\rangle=\frac{1}{\sqrt{2}}\left[|R\rangle_{1}\left|\uparrow_{z}\right\rangle_{1}|L\rangle_{2}\left|\downarrow_{z}\right\rangle_{2}-|L\rangle_{1}\left|\downarrow_{z}\right\rangle_{1}|R\rangle_{2}\left|\uparrow_{z}\right\rangle_{2}\right]
$$

" 1 " refers to particle 1 , and " 2 " refers to particle 2 , independently of what these particles 'do' even if they do the very same things (by sharing the same mixed state)—which would be impossible with descriptivism in mind. The lesson is that, if one believes that tensor product indices refer to particles, the Leibniz package cannot be saved: independently of whether one prefers the so-called 'received view' or the 'weak discernibility' approach.'

Kant also rejects the Leibniz package. He (also) rejects the PII together with individual concepts. However, Kant never talks about names or labels; instead he believes that general concepts refer to particulars when applied to intuitions. Referring via intuition-expressed in language by irreducible demonstratives ${ }^{8}$ - is a genuine alternative both to Leibniz and to the majority views in the philosophy of QM. Correspondingly, the Kantian metaphysical thesis about spatiotemporal objects is a genuine option. Both the ontology and the way of referring will (to some extent) be explained, and applied to the quantum domain, in the last section.

\footnotetext{
${ }^{6}$ Alternatively, descriptivism allows for the change of referents across possible situations (worlds; states). This would be the case with "Gödel", a label that, according to descriptivism, would refer to Schmidt in the counterfactual situation in which not Gödel but Schmidt proved the incompleteness theorem (assuming that "Gödel" carries the description of 'having proved the incompleteness theorem'). With Kripke, I take this possibility as a counter-argument against descriptivism. So, reasonable descriptivism requires essentialism concerning the relevant properties in question.

7 More on these different philosophical views about QM-objects in the following section.

${ }^{8}$ Irreducible demonstratives such as "this (here and now)..." are indexicals that cannot be expressed by (values of) variables.
} 
Fig. 3 Comparison across possible situations
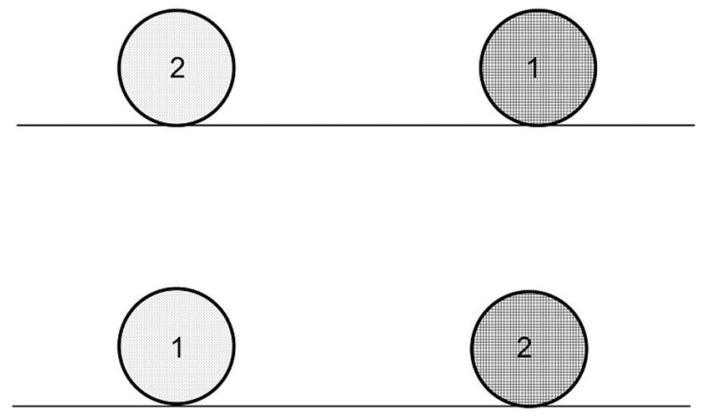

\section{Quantum Mechanics and the Labelling Problem}

There are two philosophical majority views about identity and distinguishability in QM on the market: the former Received View and the Weak Discernibility approach. According to the first, ${ }^{9}$ numerically distinct quantum particles of the same kind are, within every possible state (situation; world) and so necessarily, indistinguishable by physical properties or relations. According to the latter, ${ }^{10}$ physical particles can always be distinguished by an indeed symmetric but irreflexive physical relation, such as 'having opposite spin'.

As it seems, Weak Discernibility is a way to save the PII, at least in the sense that, necessarily, numerical distinctness goes hand in hand with physical distinguishability. Further, Weak Discernibility seems to be metaphysically 'thin', because no haecceities are required in order to distinguish the particles. In contrast to this seeming, I will argue that both majority views are essentially in the same boat: they likewise reject the PII as the principle of individuation, and both views likewise operate instead with directly referential labels. Then, both views similarly are confronted with the labelling problem, for physical reasons stemming from the distinction of purely permutation invariant states and physically entangled ones. ${ }^{11}$

The philosophical dominant views are in accordance with the straightforward reading of the standard formalism of QM according to which the tensor product indices " 1 " and "2" refer to physical particles. Nevertheless, many authors believe that something is wrong with this reading independently of any further difficulty of the philosophical views, in particular independently of the mentioned distinction of purely permutation invariant states and physically entangled ones. QM is, seemingly, confronted with a labelling problem anyway. Recall that many authors (e.g., Cortes; Dalla Chiara; French; Krause; Teller) had their worries with the tensor product indices " 1 " and " 2 " as proper names of physical particles, long before the advent of the GMW-distinction concerning (non-)entanglement.

\footnotetext{
${ }_{9}$ The seminal reference is French and Redhead (1988). According to some authors (see, e.g., Arenhart 2017), the Received View is about "non-individuals", meant in the very specific way requiring non-standard set theory. My usage of the term is in line with "the currently dominant view" according to Muller and Saunders $(2008,506)$ : "The only way to save PII is to adopt some form of thick metaphysics. Thus has arisen the currently dominant view." So, haecceitism is one option included by the Received View. Note that the main argument against the two majority views, to be presented in Section 4, goes also against the other option.

${ }^{10}$ Most prominently defended in Saunders (2006), Muller and Saunders (2008), and (also for bosons in symmetric product states) Muller and Seevinck (2009).

${ }^{11}$ More on this in the following section.
} 
However, some of them believed that (a reasonable usage of) labels must be distinguishing, or that labels must imply haecceitistic differences, or that labels must imply differences in spatial location. Thus, they did not have the right way of direct reference on board. As I will argue below, there is no such labelling problem: both majority views are safe (from the philosophy of language perspective), as long as one ignores the physical deflation of genuine entanglement.

The (alleged) labelling problem in QM may arise in the following way. The tensor product indices cannot be proper names according to descriptivism. This holds in any case, i.e. even if one ignores for a moment the requirement of permutation invariance. Non-symmetric product states are allowed then, such as (and, see again Fig. 3):

$$
\begin{aligned}
& |R\rangle_{1}\left|\uparrow_{z}\right\rangle_{1}|L\rangle_{2}\left|\downarrow_{z}\right\rangle_{2} \\
& |L\rangle_{1}\left|\downarrow_{z}\right\rangle_{1}|R\rangle_{2}\left|\uparrow_{z}\right\rangle_{2}
\end{aligned}
$$

In both states, numerically distinct particles are absolutely distinguishable so that descriptive proper names would be in order here. However, "1" and "2" cannot do that job: "1", e.g., does not refer to the particle that is located at $R$ and possesses spin-up along $z$-axis (whichever particle that may be), as the first state suggests. Instead, "1" refers to particle 1, whatever properties particle 1 may have. For, in the counterfactual situation (second state), particle 1 is located at $L$ and possesses spin-down along $z$-axis, and " 1 " still refers to that particle.

So, if the tensor product indices refer to physical particles at all, they must be proper names according to the idea of direct reference. Then, the reasoning apparently goes, such labels must carry haecceities so that every philosophical view that assumes the straightforward reading is metaphysically committed to haecceitism. Haecceitism is considered to be the metaphysical thesis according to which either there are object-involving properties on the ontological groundfloor-such as '1-ing' and '2-ing' -, or there are (fundamental) identity-involving properties such as 'being identical with particle $1 .{ }^{12}$ The direct reference approach is apparently committed to haecceitism, because Kripke's own variant- the causal theory of reference-is out of consideration with respect to QM: QM-objects do not follow spatiotemporal trajectories.

A directly referential proper name that cannot be based on a causal chain must, so the reasoning continues, carry a haecceitistic property because otherwise it cannot uniquely refer. ${ }^{13}$ In fact, in the given example of non-symmetric product states the two arrangements are qualitatively indistinguishable, but the labelling expresses a difference, namely precisely a haecceitistic difference. The labelling allows that two worlds (situations; states), exactly alike in their qualitative features - at $R$ spin up along $z$-axis and at $L$ spin-down

\footnotetext{
12 The qualification "on the ontological groundfloor" is important since, in a trivial sense, also a Russellian bundle of universals - with only universals on the ontological groundfloor-is identical to itself. The interesting haecceitistic property is a property "which is not necessarily co-extensive with any (perhaps complex) qualitative property" (Caulton and Butterfield 2012, 34). The distinction between "object-involving" and "identity-involving" is taken from Ladyman et al. (2012). A fundamental identity-involving property makes explicit that haecceitism strikes against the PII-spirit to reduce identity.

13 Strictly speaking, the non-descriptive labels carry haecceities only if the identity relation is assumed as primitive. There is disagreement in the literature about whether this requirement is substantial: compare Ladyman et al. (2012, 172) with Caulton and Butterfield (2012, 37). My argument, however, is independent of this disagreement.
} 
along $z$-axis-, may still disagree as to which object-particle 1 or particle 2-partakes in which property or relation. ${ }^{14}$

Here is my argument against this reasoning: In QM such states are forbidden. There is only one allowed state, i.e. only one possible world with these qualitative features, expressed by the running state (and, see again Fig. 2):

$$
|\psi\rangle=\frac{1}{\sqrt{2}}\left[|R\rangle_{1}\left|\uparrow_{z}\right\rangle_{1}|L\rangle_{2}\left|\downarrow_{z}\right\rangle_{2}-|L\rangle_{1}\left|\downarrow_{z}\right\rangle_{1}|R\rangle_{2}\left|\uparrow_{z}\right\rangle_{2}\right]
$$

Whatever the labels carry, there is no haecceitistic difference to be expressed, given that the particles in any actual state (world) are not absolutely qualitatively distinguishable. ${ }^{15}$ One needs two absolutely (or, at least asymmetrically) qualitatively distinguishable objects in the actual situation, and again two absolutely qualitatively objects in the counterfactual situation. Then, and only then, the question arises of whether there is a counterfactual situation that is qualitatively indistinguishable from the given actual situation but numerically distinct from it. This is the question of whether there is a haecceitistic difference. Given the Received View or Weak Discernibility, in QM, however, the presupposition can never be satisfied. Actuality is never such that there are absolutely qualitatively distinguishable objects. Haecceitistic differences, in principle, cannot occur: the labelling is harmless. ${ }^{16}$

The labelling is harmless, unless one could present a sentence that is false or meaningless simply because the reference with the tensor product indices fails. However, given the majority views, up to this point of argumentation, there is no such sentence: sentences like "particle 1 and particle 2 are both in the running state", " 1 and 2 share the same reduced mixed state", and " 1 and 2 have opposite spin" are all meaningful and true; the sentence "1 [or 2] is in such-and-such pure single-particle state" is meaningful and false, but surely not because the reference fails (but because the referent is really not in any pure single-particle state). Nothing wrong with referring, but something strange with the referents: they are interchangeable. Therefore, one needs arbitrary reference:

The solution to this issue, in my opinion, consists simply in claiming that we can fix the reference of an individual constant or variable arbitrarily. (Berto 2017, 86)

Given that the particles are necessarily not absolutely distinguishable, they are metaphysically interchangeable without any difference and so can be referred to arbitrarily. Arbitrary reference is what happens, e.g., in Existential Instantiation or Universal Generalisation: such arguments work by assuming an object 1 as an interchangeable thing satisfying a certain formula; the label "1" must be used (and can be so!) arbitrarily, otherwise the argument fails. This might be what happens according to the Received View and Weak Discernibility when one is talking about QM-objects.

So far, so good: nothing wrong with the labelling, as long as one ignores the difference between purely permutation invariant states and physically entangled ones. However, there

\footnotetext{
14 See the definition of a haecceitistic difference in Caulton and Butterfield $(2012,33)$.

15 Sometimes, philosophers argue that non-(anti-)symmetric states only are physically forbidden but metaphysically allowed. The distinction between physical and metaphysical possibilities, however, misses the point that actually there is no case of absolutely qualitative distinguishability of QM-objects. (To be precise: in this case, relatively, i.e. asymmetrically, qualitative distinguishability would suffice but is not available either).

${ }^{16}$ Here is accordance with Muller's acceptance of tensor product indices referring to physical particles (see Workshop talk).
} 
also are bad news for the defenders of Weak Discernibility: indeed, for them the PII as principle of individuation is lost. Weak Discernibility is, hence, in accordance with the following ontological view. ${ }^{17}$ On the ontological groundfloor, there are three kinds of being: (basic) particulars; properties or relations; states of affairs. The numerical distinctness of the basic particulars is irreducible, given primitively. Accordingly, they can (arbitrarily) be labelled by numbers, i.e. with non-descriptive, directly referential proper names. Primitive numerical distinctness goes with direct reference. In the QM-case, the basic ('thin') particulars can be labelled by the tensor product indices of the standard formalism. The basic states of affairs are such that the basic particulars are connected with the basic properties or relations in exactly the same way (they share the same, pure or mixed, state). Against the Received View, on the qualitative fundamental level there also are physical relations that discern the QM-objects weakly. The non-qualitative side (i.e. bare particularity), by contrast, has not been touched by Weak Discernibility. In order to go beyond this metaphysical picture a further argument is needed.

\section{The Leibnizian Solution of the Labelling Problem}

The straightforward reading according to which the tensor product indices "1" and " 2 " refer to physical particles is currently under pressure. There are two ways of banning these labels: the Leibniz strategy in favour of descriptive proper names and Kant's idea of referring via intuition. This section is devoted to the first approach.

The argument against the directly referring labels from the standard reading goes as follows. Consider again the running example:

$$
|\psi\rangle=\frac{1}{\sqrt{2}}\left[|R\rangle_{1}\left|\uparrow_{z}\right\rangle_{1}|L\rangle_{2}\left|\downarrow_{z}\right\rangle_{2}-|L\rangle_{1}\left|\downarrow_{z}\right\rangle_{1}|R\rangle_{2}\left|\uparrow_{z}\right\rangle_{2}\right]
$$

Both particle 1 and particle 2 are, as it has been said, in the same mixed state:

$$
\hat{\rho}_{1 ; 2}=\frac{1}{2}\left(\left|R, \uparrow_{z}\right\rangle\left\langle\uparrow_{z}, R|+| L, \downarrow_{z}\right\rangle\left\langle\downarrow_{z}, L\right|\right)
$$

They are, therefore, utterly indistinguishable (Received View) or discernible by a symmetric but irreflexive relation (Weak Discernibility). Now, consider the following symmetric (i.e. allowed) operator:

$$
\hat{O}=\left(\hat{R} \hat{s}_{y}\right) \otimes \hat{1}+\hat{1} \otimes\left(\hat{R} \hat{s}_{y}\right)
$$

Let it act on the given state. Apparently, at spatial location $R$ a spin-measurement in $y$-direction has been performed. The eigenvalue-eigenvector link assumed, the resulting state will be, e.g., this one:

$$
|\psi\rangle=\frac{1}{\sqrt{2}}\left[|R\rangle_{1}\left|\uparrow_{y}\right\rangle_{1}|L\rangle_{2}\left|\downarrow_{z}\right\rangle_{2}-|L\rangle_{1}\left|\downarrow_{z}\right\rangle_{1}|R\rangle_{2}\left|\uparrow_{y}\right\rangle_{2}\right]
$$

\footnotetext{
17 "In accordance" means that defenders of Weak Discernibility do not have arguments stemming from physics (or, semantics) against this_metaphysically 'thick' — view.
} 
Apparently again, the subsystem on the right side has changed its spin-property, whereas the subsystem on the left side has not been touched by the measurement. In contrast to this strong intuition, both particle 1 and particle 2 would have changed their common mixed state. They are afterwards-again not absolutely distinguishable-in this new but still common state:

$$
\hat{\rho}_{1 ; 2}=\frac{1}{2}\left(\left|R, \uparrow_{y}\right\rangle\left\langle\uparrow_{y}, R|+| L, \downarrow_{z}\right\rangle\left\langle\downarrow_{z}, L\right|\right)
$$

Although the running state is not a physically entangled state-it is "GMW-non-entangled"-, a measurement acting only at $R$ would touch and change both the particles labelled by "1" and "2". This holds likewise according to the Received View and according to Weak Discernibility; the difference between these two dominant views only is that the latter would add that both particles also have changed their (common) relation that weakly discerns them. So, both main views are, again, in the same boat: now by not doing justice to the GMW-distinction of purely permutation invariant states (as the given one) and physically entangled states. Doing justice to that distinction-and only for that reasonrequires banning the given labels.

The presented argument confirms the GMW-reading of the purely permutation invariant states, e.g.:

[T] here is a particle with spin up along z-axis and located in region $R$ and [...] there is a particle with spin down along $\mathrm{z}$-axis and located in region $L$ (Ghirardi and Marinatto 2003, 384).

There are two numerically distinct particles, in such states, which are absolutely qualitatively distinguishable, in the case at hand: one is located at $R$ with spin-up along $z$-axis, and the other is located at $L$ with spin-down along $z$-axis. These particles, however, cannot be those labelled by " 1 " and " 2 ".

If one agrees that the tensor product indices are physically empty and merely denote mathematical subspaces, one is committed to say something about how to refer to the physical particles if not by these numbers. They are, in such a case, not only numerically distinct but also absolutely qualitatively distinguishable so that it should be possible to refer to them. The first strategy is according to Leibniz, namely by introducing new, descriptive proper names: " $1_{L}$ " and " $2_{L}$ ". " $1_{L}$ " refers to the particle that possesses the properties $q_{e}, m_{e}, s=\frac{1}{2} ; R, \uparrow_{z}$ (whichever particle that may be), and " $2_{L}$ " refers to the particle that possesses the properties $q_{e}, m_{e}, s=\frac{1}{2}, L, \downarrow_{z}$ (whichever that particle may be). Given the reasoning from Section 2, one now is metaphysically committed to the PII as principle of individuation.

This commitment requires the so-called summing defence of the PII with respect to the physically entangled states. ${ }^{18}$ Take the EPR-state:

$$
|\mathrm{EPR}\rangle=\frac{1}{\sqrt{2}}\left[|\uparrow\rangle_{1}|\downarrow\rangle_{2}-|\downarrow\rangle_{1}|\uparrow\rangle_{2}\right] \otimes\left[|R\rangle_{1}|L\rangle_{2}+|L\rangle_{1}|R\rangle_{2}\right]
$$

The properties of spatial location are no longer coupled with the spin-properties. So, the only descriptive proper name that is available in order to uniquely refer to a physical object

\footnotetext{
18 See Friebe (2014, Sect. 5). Concerning symmetric product states for elementary bosons, a further defence strategy is required.
} 
refers to the system as a whole. "EPR" (say) refers to the physical object that possesses the properties $2 q_{e}, 2 m_{e}, R-L, \hat{S}^{2}=0 ; \hat{S}_{z}=0$ (whichever object that may be). In case of entanglement, the only PII-individual is an undivided whole without subsystems as parts. Disentanglement, e.g., via EPR-measurement must correspondingly be interpreted as dividing the whole, i.e. producing subsystems (particles as PII-individuals).

A challenge for this Leibniz strategy, firstly raised by Saunders (2013), ${ }^{19}$ is that, apparently, the descriptive labels are not unique, at least not in the case of fermionic non-entanglement. Every non-entangled fermion state is overdetermined in the way that it is a common eigenstate of (infinitely many) non-commuting operators. In particular, the running example state:

$$
|\Psi\rangle=\frac{1}{\sqrt{2}}\left[|R\rangle_{1}\left|\uparrow_{z}\right\rangle_{1}|L\rangle_{2}\left|\downarrow_{z}\right\rangle_{2}-|L\rangle_{1}\left|\downarrow_{z}\right\rangle_{1}|R\rangle_{2}\left|\uparrow_{z}\right\rangle_{2}\right]
$$

can also be written, e.g., in the following basis:

$$
|\Gamma\rangle=\frac{1}{\sqrt{2}}\left[|R\rangle\left|\uparrow_{z}\right\rangle+|L\rangle\left|\downarrow_{z}\right\rangle\right]|\Lambda\rangle=\frac{1}{\sqrt{2}}\left[|R\rangle\left|\uparrow_{z}\right\rangle-|L\rangle\left|\downarrow_{z}\right\rangle\right]
$$

So, according to the provided way of interpretation one apparently must argue thatalso! - there is a particle with properties associated to $|\Gamma\rangle$ and there is a particle with properties associated to $|\Lambda\rangle$.

As it seems, the joint state fails to determine its very constituents, because the mathematical physics does not tell which (sets of) distinguishing properties really are the individuating properties. In the case at hand, both $\left[|R\rangle\left|\uparrow_{z}\right\rangle\right]$ vs. $\left[|L\rangle\left|\downarrow_{z}\right\rangle\right]$ as well as $[|\Gamma\rangle]$ vs. $[|\Lambda\rangle]$ (and infinitely many more pairs of distinguishing property-sets) can do the individuating job. Responding to this challenge, one may argue that such a state never is a fundamental, i.e. a primitively given, state. It rather has been produced-namely, e.g., by dividing an entangled whole-so that the joint state taken together with the interaction that has produced it determines what the individuating properties of the particles are: ${ }^{20}$ if the dividing process is spatial-spin-like, the produced particles will be individuated by the properties of spatial location and spin, but if the dividing process is $\Gamma-\Lambda$-like, the individuating properties of the produced particles will be associated with $|\Gamma\rangle$ and $|\Lambda\rangle$.

Thus, the Leibniz strategy is tenable. However, the meaningful use of descriptive proper names referring to PII-individuals most probably requires definiteness in object number. This would restrict the Leibniz strategy to QM, inapplicable to a quantum domain with indefiniteness in particle number. This is the motivation for considering the Kantian alternative.

\footnotetext{
19 See the discussion in Caulton (2014, Sect. 7) and Bigaj (2016).

${ }^{20}$ I suggest that it is irrelevant whether the interaction takes place by a measurement or is a unitary evolution: in the latter case, the Hamiltonian determines which constituents will emerge.
} 


\section{The Kantian Solution of the Labelling Problem}

The (relevant) historical alternative to referring with individual concepts, i.e. with (comprehensively) descriptive proper names, is Kant's approach. It goes not along the straightforward reading of QM, namely not with directly referential proper names. By contrast, Kant is never talking about labels; he apparently believes that the referring problem can be solved without a theory of proper names which, in fact, is completely absent in Kant's writings. Kant's alternative is referring via intuition. It is a further alternative to the Received View and Weak Discernibility that turns out to be broader in scope than the foregoing Leibniz strategy-i.e. applicable also for the ontology of quantum field theory with delocalization and (ontological) vagueness in particle number.

Kant's approach is a variant of the metaphysical thesis that Caulton and Butterfield (2012) called "Qualitative Individuation with Indiscernibles". In Section 5 of their paper, Caulton and Butterfield discuss four metaphysical theses that result as four possible combinations of answers to two Yes-No-questions:

1 Is qualitative indiscernibility sufficient for numerical identity?

2 Is every object absolutely discerned from every other object?

The first three options have also been discussed in this paper: haecceitism (as one variant of the Received View), Weak Discernibility (as the other dominant view), and Leibnizindividuality (the first alternative, along the Leibniz-strategy performed in the foregoing section). Kant, by contrast, answers both questions in the negative: (1) qualitative indistinguishability is not sufficient for identity, (2) not every object is absolutely distinguishable from every other object; i.e. the PII cannot be saved, not even in the (deflated) sense of Weak Discernibility and not even with haecceities on the fundamental level.

According to Caulton and Butterfield, the use of (non-descriptive) labels naturally carries haecceities so that this type of metaphysics must ban the names:

Here our ban on names comes in particularly useful: according to the thesis under discussion, $x=y$ is a legitimate primitive formula of the language, representing a particular relation (namely identity); but $x=a$, which we demand be written as $\forall(y)\left(N_{a} y \equiv y=x\right)$, is illegitimate: it represents no property at all, since there are no haecceitistic properties. (Caulton and Butterfield 2012, 56) ${ }^{21}$

Correspondingly, also for Kant the primitive vocabulary of everyday language does not contain names. However, Caulton and Butterfield assume that one can instead use variables to refer to indiscernibles and to say something about them. Further, they hold that such a language is purely qualitative, i.e. one can refer to qualitative indiscernibles with a purely qualitative language. I have difficulties to understand how referring with variables might work and, also, how it can be the case that there exists a plurality of qualitative indiscernibles without a non-qualitative side. So, let us see how the Kantian alternative looks like.

According to Kant, the qualitative side of objects corresponds to the conceptual aspect of cognition. On various levels, however, Kant affirms that objects always have also a non-qualitative (but non-haecceitistic!) side, most famously in his distinction between the way in which objects appear to subjects and the way in which they are in themselves.

${ }^{21} N_{a} x$ is taken to be a haecceitistic predicate. 
Concerning the present purposes, Kant considers incongruent counterparts (e.g., left vs. right hand) and the drops of water in order to show that the conceptual aspect of cognition is not sufficient, but that also intuition is needed for perceiving or cognizing objects. The lesson is that the distinguishing capacity is captured (only) by concepts, whereas intuition (only) stands for the referring capacity. On the level of language, concepts are expressed by predicates and intuitions by irreducible demonstratives, as in the sentence: "this here and now is red".

More in detail, ${ }^{22}$ Kant rejects the PII by criticizing Leibniz to misrepresent spatiotemporal objects as "objects of pure understanding"; instead they should be "considered as appearances". ${ }^{23}$ This firstly implies that Kant does not weaken Leibniz's principle by including spatial locations/relations into the scope of the potentially distinguishing properties. Otherwise, his reproach would have been that Leibniz misrepresents the relevant properties, not the objects. Secondly, it implies that Kant does not simply introduce spatial difference as a criterion of distinguishability (but as a principle of individuation sui generis). Otherwise, it should not matter to consider the objects as appearances. ${ }^{24}$

Instead of introducing further potentially distinguishing properties or relations, Kant rejects the PII in its own way. Taking empirical objects as objects of pure understanding would mean to take them as objects for which the issue is the "comparison of concepts", i.e. to consider them as the referents of complete, i.e. individual concepts. So, what Kant essentially rejects is the philosophy of language part from Leibniz's doctrine. Considering the objects as appearances, then, cannot mean to introduce directly referential labels instead of the individual concepts. For, mere numbers would count objects independently of how they appear (to the subject), i.e. considered "in itself" (in Kant's own sense of the term). However, empirical objects are countable only considered as appearances, Kant argues. There are no bare particulars.

Thus, what Kant essentially introduces is the idea that general concepts do not refer to universals. Without being applied to intuition, they rather are 'empty', but when applied to intuition, they in fact refer to individuals. The contrast between individual concepts and general ones is hence not that the former refer to individuals, while the latter refer to universals. Instead, individual concepts refer to unique individuals from which there cannot be indiscernibles, whereas general concepts refer to merely single individuals from which there can be indiscernibles. ${ }^{25}$

The distinction between unique and merely single individuals is a modal one. Consider, as actual, one lonely drop of water: If it is a Russellian bundle of universals, then it could not have been the case that, counterfactually, there are two such drops of water. Such a bundle, i.e. a Leibniz-individual, is unique. If, alternatively, that actual drop of water has in addition to its universal properties a fundamental haecceitistic property such as '1-ing', then it could not have been the case that, counterfactually, there are two such drops of water, i.e. a second one that is also 1-ing. Haecceitism implies the uniqueness of

\footnotetext{
22 A comprehensive textual exegesis - as well as a comparison with the relevant Kant literature-can be found in Friebe (2017).

${ }^{23}$ Quotes from Kant are taken from CPR (A 263/B 319ff).

24 For, a Newtonian - not believing that space originally is pure intuition-easily can hold that spatial difference is a criterion of distinguishability.

25 Weyl (2000) calls a unique individual "Sonderwesen" (21) but a merely single individual "Einzelding" (16). So, Muller and Saunders $(2008,501)$ are wrong in their reading that Weyl is talking about individuals always in the same sense (namely, just in the first).
} 
individuals. If, contrariwise, the actual drop of water can be considered as appearance, then it is necessarily spatial (since space is the form of the appearance-corresponding intuition), and so it could have been the case that, counterfactually, there are two such drops of water, namely a second one at a different location. Objects as appearances, i.e. Kantian-individuals, are not unique but merely single entities.

This implies that, for Kant, a judgement that expresses an elementary cognition that immediately refers to its object(s) contains an irreducible demonstrative ("this here and now is red"). Such a judgement seems to be incomplete, from the perspective of formal logic. For, it contains only a predicate but not a constant, nor a bounded variable. For Kant, however, such a statement is complete, namely thanks intuition that is expressed by the irreducible demonstrative. The irreducible demonstrative does not carry any descriptionin contrast to descriptive proper names-, nor does it carry any haecceitistic property or bare particularity - in apparent contrast to directly referential proper names. It only carries space and time (as the a priori, pure part of empirical intuition), and so leads to space as the principle of individuation.

Therefore, the conceptual capacity is - and is only-distinguishing, whereas the intuitive capacity is - and is only-individuating, both in the epistemic and the ontological sense. This keeps distinguishability and individuation apart, in accordance with the rejection of the PII. It does so without introducing haecceities or bare particularity, in accordance with the Leibniz strategy. The crucial, ontological contrast between Leibniz and Kant is the distinction between the uniqueness-sense of individuality and its singleness-sense (in German: Einzigartigkeit versus Einzelnheit).

Applied to the quantum domain, the Kantian is, of course, committed to consider the position basis as privileged. ${ }^{26}$ Concerning QM, the Kantian view can be sketched state by state as follows. Consider, firstly, the running state from Fig. 2:

$$
|\psi\rangle=\frac{1}{\sqrt{2}}\left[|R\rangle_{1}\left|\uparrow_{z}\right\rangle_{1}|L\rangle_{2}\left|\downarrow_{z}\right\rangle_{2}-|L\rangle_{1}\left|\downarrow_{z}\right\rangle_{1}|R\rangle_{2}\left|\uparrow_{z}\right\rangle_{2}\right]
$$

Neither the dominant views nor the Leibniz-strategy are acceptable from the Kantian perspective. For, Kant rejects both the directly referential labels " 1 " and " 2 " from the standard reading as well as the newly introduced, descriptive labels " $1_{L}$ " and " $2_{L}$ " from the Leibnizsection. In particular, spatial locations are not properties, for Kant, but difference in space is a principle of individuation sui generis. Accordingly, spatial coordinates are mere labels, non-descriptivist ones of indistinguishable parts of space. Further, spatiotemporal objects, if one intends to label them adequately (for Kant), must be labeled via their spatial locations - in the case at hand: " $R$ " and " $L$ ". So, the Kantian reading of the given state is: " $R$ shows spin-up along $\mathrm{z}$-axis, and $L$ shows spin-up along z-axis."

Now, take a physically entangled state, such as the EPR-state:

$$
|\mathrm{EPR}\rangle=\frac{1}{\sqrt{2}}\left[|\uparrow\rangle_{1}|\downarrow\rangle_{2}-|\downarrow\rangle_{1}|\uparrow\rangle_{2}\right] \otimes\left[|R\rangle_{1}|L\rangle_{2}+|L\rangle_{1}|R\rangle_{2}\right]
$$

Again, the Leibniz-strategy to consider the (allegedly composite) whole as undivided and to label it with the newly introduced, descriptive proper name "EPR" - that carries the

\footnotetext{
${ }^{26}$ This can-perhaps — be done with the decoherence approach. It is not intended to consider the Bohmian alternative.
} 
description of the properties $2 q_{e}, 2 m_{e}, R-L, \hat{S}^{2}=0 ; \hat{S}_{z}=0$-is not available for the Kantian. However, probably the Kantian strategy is closer to GMW's original description of the situation. Accordingly, in such a state

it is not possible, for example, to attribute any definite spin property to the particle located in $R$ and equivalently no definite spatial property can be attributed to the particle with spin up (Ghirardi and Marinatto 2003, 384).

Together with privileging the position basis, one should say that in fact there is a particle located in $R$, and that there is a second particle at $L$. Both particles can be considered by the Kantian in the same way as in the case of non-entanglement.

The problem seems to be the indefiniteness of their spin properties. However, note that the requirement of definiteness of property attribution does not follow from Kant's way of rejecting the PII. Kant's concept of a merely single individual does not strike against indefiniteness of properties; it strikes (only) against the uniqueness of individuals, i.e. against Leibniz-individuality and against haecceitism. In the case at hand, one has one merely single object at $R$ and one other merely single object at $L$ : regardless of the properties either objects may have, definitely or not.

The hard problem, for the Kantian, consists in states such as the following:

$$
|\psi\rangle=\frac{1}{\sqrt{2}}|R\rangle\left[|\uparrow\rangle_{1}|\downarrow\rangle_{2}-|\downarrow\rangle_{1}|\uparrow\rangle_{2}\right]
$$

Both (alleged) particles, 1 and 2, are located in the same region $R$. According to this standard reading, they are numerically different independently of their spatial location, which clearly goes against Kant's view of space as the principle of individuation. This is the case in which the Kantian must apply the summing defence and claim: there is only one lonely - but merely single - individual with twice the mass and twice the charge of an elementary particle, and with spin-projection properties equal zero. ${ }^{27}$

The advantage over the Leibniz strategy is that the Kantian view can be extended to quantum field theory. Whereas the uniqueness-sense of individuality requires $n$ unique particles or one unique field, the singleness-sense of individuality is open for dislocation, variability, and indefiniteness in particle number. ${ }^{28}$ The question, raised by quantum field theory, of whether there are precisely $n$ particles, or only one lonely field spread out in the whole of space(time), or indefinitely many, more or less localizable entities, is conceived of as an empirical, not as an ontological question. ${ }^{29}$ Understood in the Kantian way, it concerns the distinguishing part of cognition, i.e. the predicative part of the judgement "this here and now is [...]", as in the cases: "this here and now is delocalized" or "this here and now is indefinite in number". The formal way of thinking, i.e. the formal structure of empirical judgements- 'irreducible demonstrative plus predicate'-, which expresses the ontology of merely single individuals, still is the same as in QM.

Shortly summarized, Kant's claim that both intuition and concepts are needed for and play a significantly distinctive role in cognition means, applied to the current context, that the non-qualitative side of empirical objects can be saved without directly

\footnotetext{
27 Further work is needed here in order to justify this variant of the summing defence.

${ }^{28}$ Number is now an operator. The system can be in various eigenstates of this operator, but also in a superposition of them.

29 Number is now an operator!
} 
referential numbers and so without haecceities. This is to say that the individuality of objects can so be saved, namely in the singleness-sense. In this way, counting the objects becomes merely empirical, i.e. not metaphysical, as it were with haecceities or with unique (essential) qualitative properties. Empirically, the number of objects may be indefinite, which does not imply that the empirical world does not have a non-qualitative side.

\section{Conclusion}

The two dominant views in the philosophy of physics-Received View and Weak Discernibility - have been challenged by focusing on the distinction between purely permutation invariant states and physically entangled ones. This requires to ban the directly referential labels used according to the standard reading of QM and to investigate alternative ways of referring in the quantum domain (connected with alternative ontologies).

Two different alternatives to direct reference have been considered: the Leibnizian strategy of referring with (comprehensively) descriptive proper names to PII-individuals, i.e. to objects (particles) individuated by distinguishing absolute and qualitative properties, and the Kantian strategy of referring without any label but with help of intuition to merely single individuals. It has been argued that the Kantian way is broader in scope than the Leibnizian.

Acknowledgements Open Access funding provided by Projekt DEAL. Thanks to Tomasz Bigaj and two anonymous Referees for their fruitful comments.

Open Access This article is licensed under a Creative Commons Attribution 4.0 International License, which permits use, sharing, adaptation, distribution and reproduction in any medium or format, as long as you give appropriate credit to the original author(s) and the source, provide a link to the Creative Commons licence, and indicate if changes were made. The images or other third party material in this article are included in the article's Creative Commons licence, unless indicated otherwise in a credit line to the material. If material is not included in the article's Creative Commons licence and your intended use is not permitted by statutory regulation or exceeds the permitted use, you will need to obtain permission directly from the copyright holder. To view a copy of this licence, visit http://creativecommons.org/licenses/by/4.0/.

\section{References}

Arenhart, J. R. B. (2017). The received view on quantum non-individuality: Formal and metaphysical analysis. Synthese, 194, 1323-1347.

Berto, F. (2017). Counting the particles: Entity and identity in the philosophy of physics. Metaphysica, $18(1), 69-89$.

Bigaj, T. (2016). On some troubles with the metaphysics of fermionic compositions. Foundations of Physics, 46(9), 1168-1184.

Caulton, A., \& Butterfield, J. (2012). On kinds of indiscernibility in logic and metaphysics. British Journal for the Philosophy of Science, 63(1), 27-84.

Caulton, A. (2014). Qualitative individuation in permutation-invariant quantum mechanics. arXiv: $1409.0247 \mathrm{v} 1$ [quant-ph].

French, S., \& Redhead, M. (1988). Quantum physics and the identity of indiscernibles. The British Journal for the Philosophy of Science, 39(2), 233-246.

Friebe, C. (2014). Individuality, distinguishability, and (non-)entanglement: A defence of Leibniz's principle. Studies in History and Philosophy of Modern Physics, 48(A), 89-98.

Friebe, C. (2017). Kant's rejection of Leibniz's principle and the individuality of quantum objects. Kant Yearbook, 9, 1-17. 
Ghirardi, G., Marinatto, L., \& Weber, T. (2002). Entanglement and properties of composite quantum systems: A conceptual and mathematical analysis. Journal of Statistical Physics, 108, 49-122.

Ghirardi, G., \& Marinatto, L. (2003). Entanglement and properties. Fortschritte der Physik, 51, $379-387$. Kant, I. (1781/87). Critique of Pure Reason (CPR), A- and B-edition.

Ladyman, J., Linnebo, O., \& Pettigrew, R. (2012). Identity and discernibility in philosophy and logic. The Review of Symbolic Logic, 5(1), 162-186.

Muller, F. A., \& Saunders, S. (2008). Discerning fermions. British Journal for the Philosophy of Science, 59(3), 499-548.

Muller, F. A., \& Seevinck, M. P. (2009). Discerning elementary particles. Philosophy of Science, 76(2), 179-200.

Rodriguez-Pereyra, G. (2014). Leibniz's principle of identity of indiscernibles. Oxford: Oxford University Press.

Saunders, S. (2006). Are quantum particles objects? Analysis, 66, 52-63.

Saunders, S. (2013). Indistinguishability. In R. Batterman (Ed.), Oxford handbook of philosophy of physics (pp. 340-380). Oxford: Oxford University Press.

Weyl, H. (2000). 1928; 1949. Philosophie der Mathematik und Naturwissenschaft. München.

Publisher's Note Springer Nature remains neutral with regard to jurisdictional claims in published maps and institutional affiliations. 